\title{
Ergonomic Evaluation of Current Advancements in Blueberry Harvesting
}

\author{
Eunsik Kim ${ }^{1} * * \mathbb{C}$, Andris Freivalds ${ }^{2}$, Fumiomi Takeda ${ }^{3}$ and Changying $\mathrm{Li}^{4}$ \\ 1 Department of Industrial \& Manufacturing Systems Engineering, University of Windsor, \\ Windsor, ON N9B 3P4, Canada \\ 2 Harold and Inge Marcus Department of Industrial and Manufacturing Engineering, The Pennsylvania State \\ University, State College, PA 16802, USA; axf@engr.psu.edu \\ 3 United States Department of Agriculture-Agricultural Research Service, Appalachian Fruit Research \\ Station, Kearneysville, WV 25430, USA; fumi.takeda@ars.usda.gov \\ 4 College of Engineering, University of Georgia, Athens, GA 30602, USA; cyli@uga.edu \\ * Correspondence: eskim@uwindsor.ca; Tel.: +1-519-253-3000 (ext. 5409)
}

Received: 23 October 2018; Accepted: 14 November 2018; Published: 17 November 2018

\begin{abstract}
Work-related musculoskeletal disorders (MSDs) accounted for $32 \%$ of days-away-from-work cases in private industry in 2016. Several factors have been associated with MSDs, such as repetitive motion, excessive force, awkward and/or sustained postures, and prolonged sitting and standing, all of which are required in farm workers' labor. While numerous epidemiological studies on the prevention of MSDs in agriculture have been conducted, an ergonomics evaluation of blueberry harvesting has not yet been systematically performed. The purpose of this study was to investigate the risk factors of MSDs for several types of blueberry harvesting (hand harvesting, semi-mechanical harvesting with hand-held shakers, and over-the-row machines) in terms of workers' postural loads and self-reported discomfort using ergonomics intervention techniques. Five field studies in the western region of the United States between 2017 and 2018 were conducted using the Borg CR10 scale, electromyography (EMG), Rapid Upper Limb Assessment (RULA), the Cumulative Trauma Disorders (CTD) index, and the NIOSH (National Institute for Occupational Safety and Health) lifting equation. In evaluating the workloads of picking and moving blueberries by hand, semi-mechanical harvesting with hand-held shakers, and completely mechanized harvesting, only EMG and the NIOSH lifting equation were used, as labor for this system is limited to loading empty lugs and unloading full lugs. Based on the results, we conclude that working on the fully mechanized harvester would be the best approach to minimizing worker loading and fatigue. This is because the total component ratio of postures in hand harvesting with a RULA score equal to or greater than 5 was $69 \%$, indicating that more than half of the postures were high risk for shoulder pain. For the semi-mechanical harvesting, the biggest problem with the shakers is the vibration, which can cause fatigue and various risks to workers, especially in the upper limbs. However, it would be challenging for small- and medium-sized blueberry farms to purchase automated harvesters due to their high cost. Thus, collaborative efforts among health and safety professionals, engineers, social scientists, and ergonomists are needed to provide effective ergonomic interventions.
\end{abstract}

Keywords: blueberry harvesting; work-related musculoskeletal disorders; ergonomics intervention

\section{Introduction}

Work-related musculoskeletal disorders (MSDs) accounted for 32\% (285,950 cases) of days-away-from-work cases in private industry in 2016 and occurred at a rate of 29.4 cases per 
10,000 full-time equivalent workers [1]. The term musculoskeletal disorder refers to injuries and disorders of the locomotor apparatus, i.e., muscles, tendons, bones, cartilage, blood vessels, ligaments, and nerves. Work-related musculoskeletal disorders (MSDs) include all musculoskeletal disorders that are induced or aggravated by work and the circumstances of its performance. These painful and often disabling injuries generally develop gradually over weeks, months, and years. According to the National Institute for Occupational Safety and Health (NIOSH), several epidemiological studies have demonstrated evidence of a causal relationship between physical exertion at work and work-related musculoskeletal disorders (MSDs) [2].

MSDs have been associated with repetitive motion, excessive force, awkward and/or sustained postures, and prolonged sitting and standing, all of which are required in farm workers' labor. Furthermore, workers are often paid on a piece-rate system, providing an incentive to work at high speed and skip recommended breaks. This results in labor-intensive practices and high rates of musculoskeletal disorders among farmers and farm workers [3-7].

While numerous epidemiological studies on the prevention of MSDs in Agriculture have been conducted [4,6,8-10], an ergonomics evaluation of manual blueberry harvesting has not yet been systematically performed. The highbush blueberry industry in the United States has experienced rapid growth in the past three decades, producing 231 megatons of blueberries per year, accounting for about $57 \%$ of the annual production of highbush blueberries worldwide [11]. Furthermore, as consumers become increasingly aware of healthful eating and of blueberries' convenience, flavor, and ease of consumption in various snacks, salads, and baking, North America, including the U.S., Canada, and Mexico, will continue to have the most developed fresh blueberry market in the world, as shown in Figure 1.

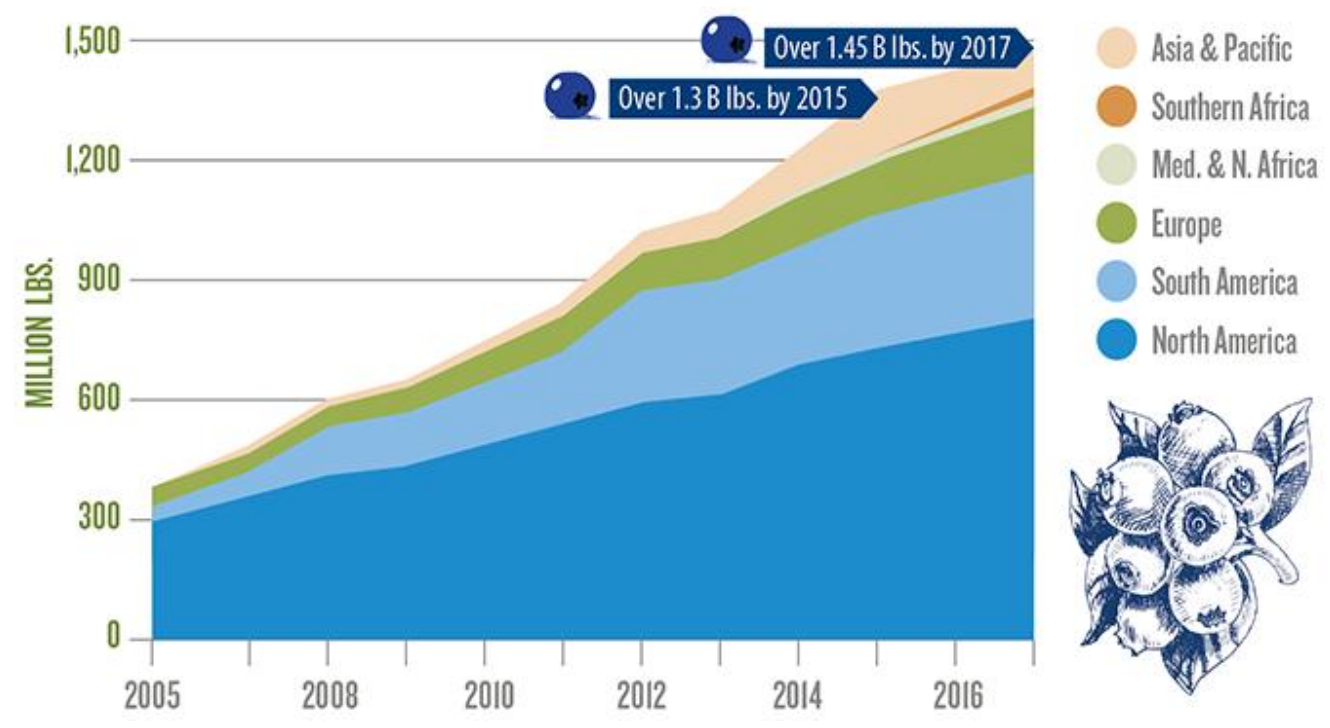

Figure 1. World highbush blueberry production: Growth predictions (source: Cort Brazelton-North American Blueberry Council 2014. Reproduced with permission from North American Blueberry Council).

With such rapid growth in the production and consumption of blueberries in the last 10 years, there has been an increased need for laborers to maintain the bushes and harvest the fruit. These workers are exposed to a high musculoskeletal workload caused by weight, work posture, and repetitive motion. Although over-the-row (OTR) mechanical harvesters are available on the market, much of the blueberry harvesting is still done by hand, since the cost of OTR machines is too high for small- and medium-sized blueberry farms, and since blueberries harvested with OTR machines are likely to be bruised or damaged, making them un acceptable for extended cold storage and the long transport to distant consumers [12,13]. 
Previous studies on the harvesting of blueberries have focused on harvest efficiency and the quality and quantity of blueberries rather than the labor conditions of the farm worker. In order to improve harvest efficiency, several researchers have sought to develop and evaluate new methods for harvesting blueberries by comparing hand harvesting and semi-machine harvesting with hand-held shakers and OTR machines $[11,14,15]$. An ergonomic analysis of new machines for mechanical harvesting with a lower cost option (e.g., harvesting blueberries from the ground with hand-held pneumatic shakers and catching fruit using a portable catching frame) was recently described by Takeda et al. [11]. The purpose of this study was to investigate the risk factors of MSDs for each type of blueberry harvesting method (hand-picking, semi-mechanical harvesting, and handling of trays on fully mechanized harvesters) in terms of workers' postural loads and self-reported discomfort using ergonomics intervention techniques.

\section{Materials and Methods}

We conducted five field studies in three western states (California, Oregon, and Washington) of the United States between 2017 and 2018. Since some companies only permitted data collection via video recording and direct observation, we did not have access to such detailed demographic information as age, education level, smoking status, work experience, and anthropometry data for all participating workers. For hand harvesting, five measurements were used to evaluate workload. First, muscle activity was measured using electromyography (EMG) (Thought Technology Ltd, Montreal West, QC, Canada). The electrode sensors were attached to participants' arms, shoulders, and lower back (Figure 2). Second, Rapid Upper Limb Assessment (RULA) was conducted to estimate the postural risks of work-related upper limb injury. Third, subjective perceived exertion was measured with a Borg CR10 scale to evaluate the workload of harvesting blueberries on the neck, shoulder, arm, hand, and low back. Fourth, the NIOSH lifting equation was used to calculate the risk factor of lifting. Finally, the Cumulative Trauma Disorders (CTD) index was used to compute the potential risk for cumulative trauma disorders caused by repetitive movement. For semi-machine harvesting with hand-held shakers, four measurements were used to evaluate workloads, including EMG, RULA, CTD index, and the NIOSH lifting equation. Lastly, for the completely mechanized harvesting system, only EMG and the NIOSH lifting equation were used, as labor for this system is limited to loading empty lugs and unloading full lugs.

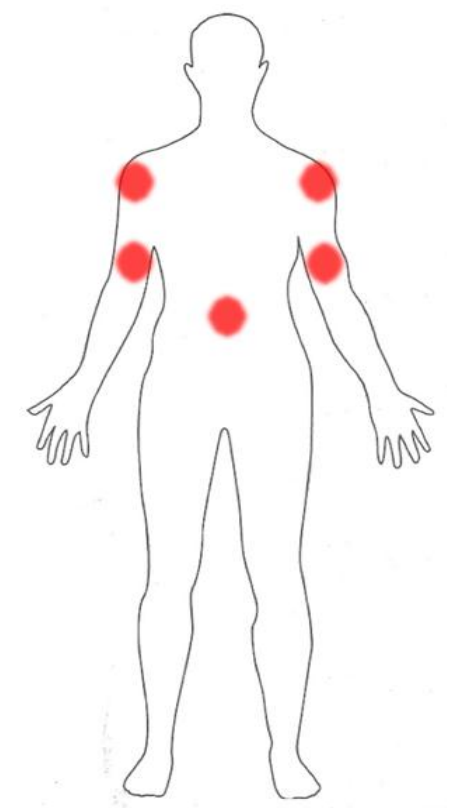

Figure 2. Muscle activity measurement map. 


\subsection{Blueberry Harveating Method}

Highbush blueberries destined for the fresh market have been harvested by hand to maximize quality and postharvest shelf-life. In contrast, nearly all blueberries destined for the process and frozen market is harvested by over-the-row (OTR) machines such as the Oxbo 7440 berry harvester (Oxbo International, Lynden, WA, USA). However, many growers have reported difficulty obtaining sufficient labor for hand harvest operations and the cost of labor is steadily increasing. As a result, some blueberries sold on the fresh market are now harvested by OTR machines, in which fruit is detached with fully mechanized shakers and the workers on these machines handle flats, fill them with berries rolling off the conveyor belt, and then stack filled flats onto a pallet. The third option for harvesting blueberries is to use hand-held, pneumatic, and electric shakers and collect detached berries using a portable catch frame [11] or mechanized fruit conveyance system.

\subsubsection{Hand Harvesting}

Blueberries do not ripen simultaneously in the cluster. The workers harvesting blueberries by hand wear a shoulder or waist belt harness on which a pail or bucket can be hooked. When one or more berries in a cluster become ripe, the workers clasp the cluster with one or both hands with the palm of the hand underneath the cluster. Ripe berries are teased off the cluster by rolling the thumb or the index finger over them individually until they detach from the pedicel. When several berries are collected in the palms of the hands, the fruit is gently placed in the container. A worker normally picks about $25-30 \mathrm{~kg}$ per hour, and expert pickers pick up to $50 \mathrm{~kg}$ per hour and work about $6-7 \mathrm{~h}$ per day. A flat or pail of blueberries weighs about 7 to $10 \mathrm{~kg}$, depending on the size of box. Thus, each worker picks between 20 and 50 pails per day. When the containers are filled with blueberries, the workers lift, lower, or carry them to the weighing station located at the edge of the field. Depending on the length of the row, workers walk out of the field carrying three or four containers, each weighing between 7 and $10 \mathrm{~kg}$, for as much as $200 \mathrm{~m}$ (see Figure 3). Once the fruit is weighed and transferred to flats, workers carry the empty picking containers back through the row to harvest more blueberries. Examples of carrying postures are shown in Figure 3. The total number of carrying trips can be between 10 and 20 times per day for each worker.

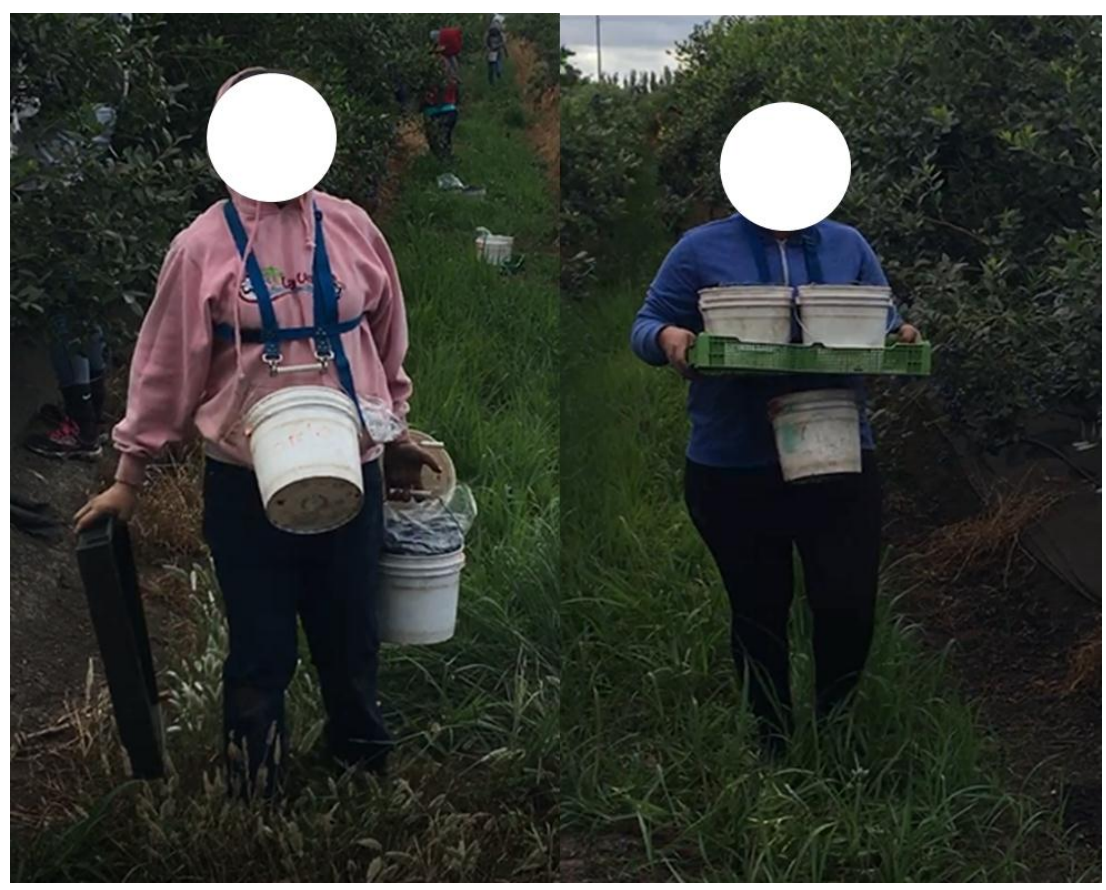

Figure 3. Examples of carrying posture. 


\subsubsection{Semi-Mechanical Harvesting with Hand-Held Shakers}

For the semi-mechanical harvesting method, a long-handled, pneumatically-operated olive harvester (Campagnola, Bologna, Italy) was used to harvest blueberries from a platform on a modified over-the-row harvester (Model 7240, Oxbo International, Lynden, WA, USA). The olive harvester (10) consisted of a 1.2-m-long aluminum tube with a grip in the mid-section and the trigger handle grip at the end. At the other end of the tube, an attachment consisting of an air motor/piston housing to which two reciprocally moving heads with four $20-\mathrm{cm}$-long plastic tines were mounted. The tips of tines had a lateral displacement of about $5 \mathrm{~cm}$. The speed at which the air motor rotated and actuated the piston's in-and-out motion, which caused the two heads to move left and right, was controlled with air pressure. For this study, the olive shakers were operated at about $450 \mathrm{KPa}$, which caused the heads with tines to move back and forth at $\sim 1.2 \mathrm{~Hz}$. Workers held the olive shaker with two hands with the hand at the opposite end from the shaker head controlling the on/off trigger. The long handle allowed workers to operate the shaker in the standing position with the handle oriented horizontally across at waist to chest height. It was also possible to raise the shaker head even above the worker's head to harvest blueberries at the top of 2-m-tall blueberry plants or to lean forward or bend the knees and point the shaker downward to harvest blueberries located close to the ground.

\subsubsection{Over-the-Row Machine Harvesting (OTR)}

Over-the-row blueberry harvesters are large machines designed to travel over rows of blueberry plants, one row at a time [11]. They typically have an inverted " $U$ "-shaped frame tunnel large enough to move over large blueberry plants with sufficient clearance at the top. For this study, two harvesters (Oxbo 7440 and 8040, Oxbo International, Lynden, WA, USA) were used. Both harvesters detach blueberries with a rotary shaker mounted on each side of the tunnel. In both machines, detached berries are collected by catch plates on each side of the tunnel and roll onto a conveyor belt on each side of the harvester. Model 8040 is a single-drop harvester which transfers the fruit through a cleaning system to the back of machine, where it falls into a flat placed on a shelf. There are two workers on the platform on each side of the harvester. The first worker places an empty flat on the shelf underneath the end of the conveyor belt, waits for the flat to fill with blueberries, stops the belt by closing the hydraulic line, removes the filled flat from the shelf, and hands it to a second worker. The second worker takes the flat and stacks. Model 7440 is a top-load berry harvester designed to handle high fruit volume. The harvested fruit rolls onto a conveyor belt on each side of the harvester that moves the fruit to the back, where the fruit is transferred to an elevator bucket that moves the fruit to the platform at the top of the harvester. There the fruit is transferred to a horizontal conveyor belt, first going under a leaf/trash suction blower. The cleaned fruit is then transferred to another belt which moves the fruit horizontally. At the end of the conveyor belts, the fruit drops into a flat. Workers stationed on the top platform move empty flats that are stacked on pallets, and places them under the end of the conveyor belt to collect falling fruit in the flat. When the flat is full then the first worker lifts the filled flat and hands it to another worker. This worker then moves the filled flat to another pallet for stacking. There are shallow and deep flats used to collect machine-harvested blueberries. Shallow flats weigh about $4 \mathrm{~kg}$ when filled with blueberries. Deep flat weights $7 \mathrm{~kg}$ when filled with blueberries. For shallow trays, workers make six columns of flats on a pallet. As many as 12 flats are stacked, reaching a height of $1.4 \mathrm{~m}$. When deep trays are used, workers also create six columns and the filled flats are stacked eight high, reaching a height of $1.8 \mathrm{~m}$.

\subsection{Ergonomics Intervention Techniques}

\subsubsection{Borg Category Ratio Scale (CR10)}

The Borg category ratio scale (CR10) was used to determine workers' health and discomfort at work [16]. Borg CR10 is a subjective scale in which participants self-rate their level of physical pain or discomfort for various parts of the body on a scale from 0 to 10 , where 0 represents no pain at all and 
10 represents maximum pain. In the literature, numerous studies have validated the Borg scale with quantitative measurements of physiological responses (e.g., metabolic acidosis, ventilation, oxygen intake, heart rate, and respiration frequency) [17-19].

\subsubsection{Rapid Upper Limb Assessment (RULA)}

McAtamney and Corlett [20] developed the Rapid Upper Limb Assessment (RULA) to examine the level of risk of upper limb disorders for individual workers. RULA evaluates worker exposure to the position, force, and repetitive movement of different work postures that contribute to repetitive strain injuries (RSIs). It is a widely used measurement because it is easy and provides a quick assessment without requiring special equipment. RULA encompasses the postures of several body parts, including the wrists, arms, neck, shoulders, trunk, and legs, and accounts for force as well as repetition in those postures. The assessment consists of two sections, Section A and Section B. In Section A, scores are entered for the shoulders, arms, and wrists, while scores for the legs, neck, and trunk are entered in Section B. The posture scores from Sections A and B are then combined with the muscle use score and force score to obtain the grand score, which represents the level of MSD risk. The MSD risk score ranges from 1 to 7 , where a score of 1 or 2 indicates an acceptable risk, 3 or 4 indicates low risk and may warrant further investigation, 5 or 6 indicates medium risk and requires investigation and change, and a score of 7 indicates high risk and requires immediate investigation and change.

\subsubsection{Cumulative Trauma Disorders (CTD) Risk Assessment Model}

The original version of the Cumulative Trauma Disorders (CTD) risk assessment model assesses the risk of CTDs in the upper extremities [21]. A simplified version of the CTD risk index appears in Niebel and Freivalds [22]. This version reduces the analysis complexity and time required for the assessment and is therefore more appropriate for evaluation in field study. This version assesses four factors: frequency, posture, force, and miscellaneous. The frequency factors index is calculated based on the number of hand motions per day scaled by the allowable limit of 10,000 daily hand motions. The posture factor is determined from grip types and the degree of deviation from the natural posture of the upper limbs. The force factor index is calculated based on percent maximum voluntary contraction (MVC) used in a given task and then scaled by 15 percent. Miscellaneous factors include the use of gloves, the presence of sharp edges on work contact surfaces, vibration exposure, and cold temperatures. These four factors are then weighted and summed to obtain a final CTD risk index as a job risk measure with a critical value of 1 .

\subsubsection{NIOSH Lifting Analysis}

The National Institute for Occupational Safety and Health (NIOSH) published the Revised NIOSH lifting equation for evaluating the physical demands of two-handed manual lifting tasks based on biomechanical, psychophysical, physiological, and epidemiological factors in 1993 [23]. The NIOSH lifting equation has been used in several research studies to quantify biomechanical stressors from manual lifting and lowering tasks and has become the most commonly used job analysis method in the past two decades [24-27]. The lifting equation consists of two steps: (1) calculate the recommended weight limit (RWL, i.e., the maximum acceptable load), and (2) calculate the lifting index (LI, i.e., the relative estimate of the level of physical stress and MSD risk associated with lifting tasks) for a specified manual lifting task. The RWL is calculated depending on lifting conditions, e.g., hand location in relation to the body, vertical travel distance of hands, degree of symmetry in posture, frequency of lifting, work-rest duration pattern, and type of hand coupling:

$$
\left.R W L=51 \times\left(\frac{10}{H}\right) \times(1-0.0075 \times|V-30|) \times\left(0.82 \times \frac{1.8}{D}\right) \times F M \times 1-0.0035 \times A\right) \times C M
$$


where the factors in the equation are:

$H=$ horizontal load distance,

$V=$ vertical load distance,

$D=$ vertical displacement of the load,

$F M=$ frequency multiplier,

$A=$ asymmetric factor, and

$C M=$ coupling multiplier.

$L I$ is the ratio of the current load weight to the recommended load weight limit:

$$
L I=\frac{\text { Load Weight }}{R W L}
$$

An $L I$ value of less than 1.0 indicates safe lifting without an increased risk of low back pain (LBP). An $L I>1$ has been shown to be associated with LBP in previous studies [28,29]. Thus, the goal is to design all lifting jobs such that they result in an $L I$ value of less than 1.0.

\subsection{Video Recording Observation and Data Processing}

Twelve farm workers (male: 11, female: 1) were observed during the harvesting process via video recording. For each worker, five-minute samples were recorded at randomly selected periods during the harvesting process. The video was captured at one-second intervals. About 3000 images per worker were captured. These images were categorized into groups for posture analysis. Figure 4 shows the example of categorized harvesting postures and the component ratio of each posture group. In Figure 4, the postures are defined as follows: for hand harvesting, high (H-A), middle (H-B), low (H-C), stretch (H-D), and squat (H-E); for semi-machine harvesting, push in high position (M-A), pull in high position (M-B), push in low position (M-C), pull in high position (M-D), and standing (M-E). EMG signals were obtained using disposable surface electrodes (Thought Technology TTL T3404; an active diameter of $1.0 \mathrm{~cm}$ and an inter-electrode distance of $2.4 \mathrm{~cm}$ ). The electrodes were placed in the direction of the muscle fibers on the worker's skin after standard skin preparation. The EMG signals of each muscle were amplified and automatically converted into root mean square (RMS) values via a MyoScan-Pro sensor (Thought Technology Ltd, Montreal West, QC, Canada). The EMG signals for each participant's biceps brachialis (BB), anterior deltoid (AD), and L5 level on the trunk were normalized by:

$$
E M G_{n, m}(\% M V C)=\frac{E M G_{t a s k}}{E M G_{\max }} \times 100
$$

where $E M G_{n, m}$ stands for the RMS EMG of each muscle for each participant and $E M G_{\text {max }}$ is the maximum RMS EMG signal of each muscle obtained for all recorded postures for each participant. $E M G_{\text {task }}$ represents the actual electromyographic activity of a specific muscle during the blueberry harvestings. 


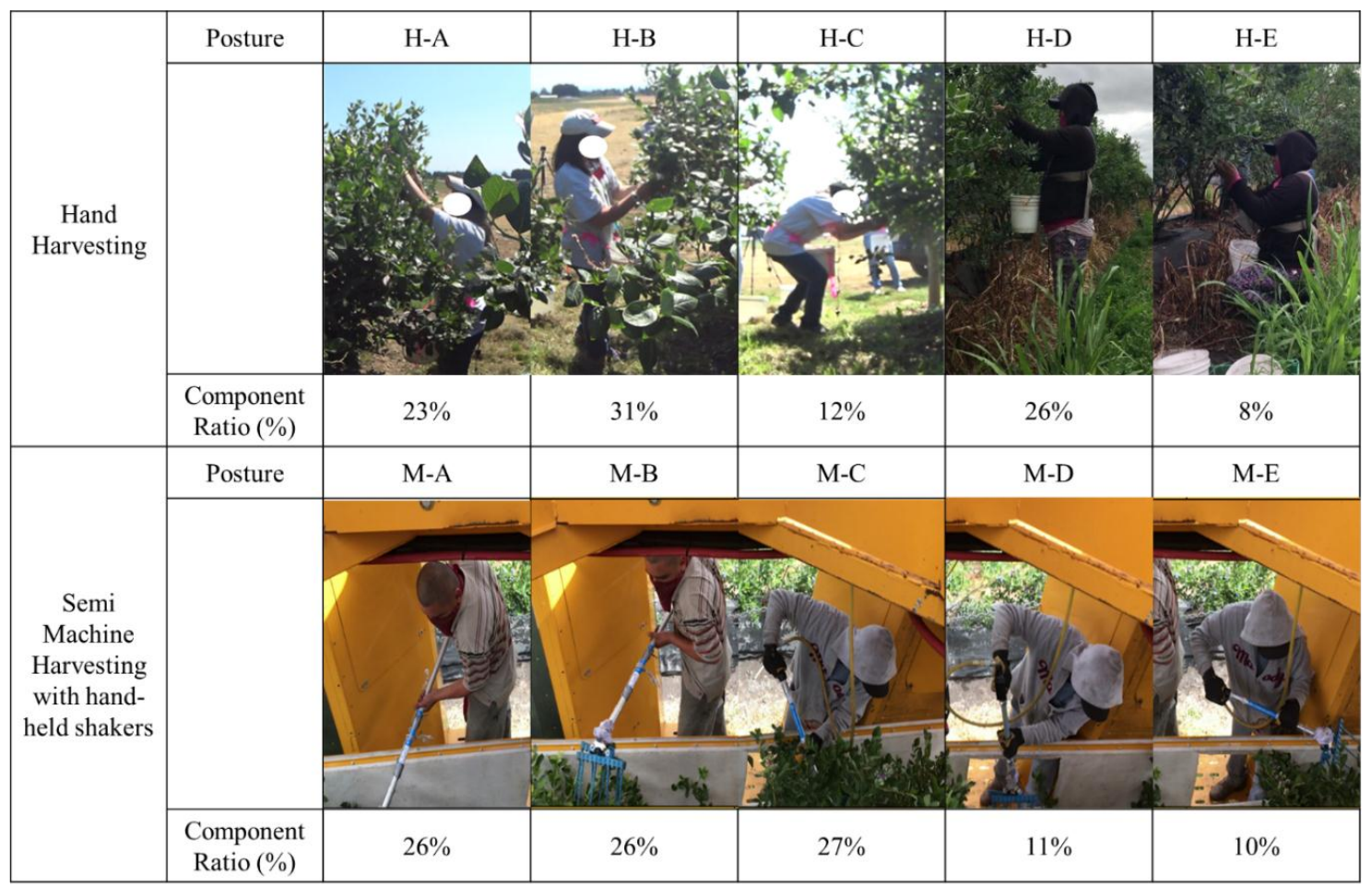

Figure 4. Examples of categorized harvesting postures and component ratios of each posture group: For hand harvesting, high (H-A), middle (H-B), low (H-C), stretch (H-D), and squat (H-E); for semi-machine harvesting, push in high position (M-A), pull in high position (M-B), push in low position (M-C), pull in high position (M-D), and standing (M-E).

\section{Results}

\subsection{Demographic Result}

Half of the subjects did not exercise regularly, and more than five had low education levels (i.e., through lower secondary school). Details of the blueberry harvester demographic data are shown in Table 1.

Table 1. Demographic information (SD = Standard deviation).

\begin{tabular}{ccc}
\hline \multicolumn{2}{c}{ Characteristics } & N (\%) \\
\hline \multirow{2}{*}{ Gender } & Male & 11 \\
& Female & 1 \\
\hline \multicolumn{2}{c}{ Age in years (mean \pm SD) } & $31.3 \pm 15.8$ \\
\hline \multirow{2}{*}{ Educational level } & Primary school & 5 \\
& Lower secondary school & 4 \\
& Upper secondary school & 3 \\
\hline \multirow{2}{*}{ Smoking Status } & Yes & 3 \\
& No & 9 \\
\hline \multicolumn{2}{c}{ Years smoking (mean \pm SD) } & 6 \\
\hline \multirow{2}{*}{ Exercise frequency } & None & 3 \\
& Some & 3 \\
\hline
\end{tabular}

On average, each blueberry farm laborer had worked 6.9 years at a rate of $8.3 \mathrm{~h}$ per day with a total of 17.9 min spent on breaks. Our analysis showed that half of the subjects experienced fatigue during daily work activities, and that most experienced the highest level of discomfort in the shoulder 
area. Table 2 shows the blueberry harvesters' physical ergonomic factors in their labor based on the questionnaire.

Table 2. Physical ergonomic factors of worker ( $n=12, \mathrm{SD}=$ Standard deviation).

\begin{tabular}{ccc}
\hline \multicolumn{2}{c}{ Characteristics } & N (\%) \\
\hline Working experience $($ mean \pm SD) years & $6.9 \pm 7.5$ & \\
\hline Working days in a month (mean \pm SD) days & $26 \pm 6.3$ & \\
\hline Working hours in a day $($ mean \pm SD) hours & $8.3 \pm 1.6$ & \\
\hline Break duration (mean \pm SD) minutes & $17.9 \pm 9.9$ & \\
\hline \multirow{2}{*}{ Experiencing work fatigue } & Yes & 6 \\
Frequency of work fatigue & No & 6 \\
\hline & Sometimes & 6 \\
& Frequently & 5 \\
& Always & 1 \\
\hline \multirow{2}{*}{ Body parts with greatest discomfort } & Shoulder & 8 \\
& Arm & 4 \\
& Hand & 2 \\
& Low back & 6 \\
& Leg & 6 \\
\hline
\end{tabular}

\subsection{Hand Harvesting}

Regarding CTD risk assessment, both the mean of the frequency factor of 2.24 (SD: 0.12) and the posture factor of 1.43 (SD: 0.06) exceeded the safety threshold of 1.0, leading to a total risk value mean of 1.30 (SD: 0.05), which also exceeded 1.0. Table 3 shows the RULA scoring during blueberry harvesting. Score A represents wrist and arm scores and Score B refers to neck, trunk, and leg scores. Based on a Score A of 6 and a Score B of 7, posture H-A showed a RULA score of 7, the highest score among the evaluated postures, indicating that this harvesting posture needs further analysis and that immediate change should be implemented. Posture B displayed a RULA score of 3, the lowest RULA score among the evaluated postures, indicating that this harvesting posture needs to be changed or performed less frequently. Overall, the total component ratio of postures with a RULA score equal to or greater than 5 was $69 \%$, indicating that more than half of the postures used during hand harvesting were medium risk and called for engineering and/or work method changes to reduce or eliminate MSD risk.

Table 3. Rapid Upper Limb Assessment (RULA) analysis results.

\begin{tabular}{ccccc}
\hline Posture & Component Ratio (\%) & Score A & Score B & Grand Score \\
\hline H-A & 23 & 6 & 7 & 7 \\
H-B & 31 & 4 & 3 & 3 \\
H-C & 12 & 6 & 5 & 6 \\
H-D & 26 & 6 & 3 & 5 \\
H-E & 8 & 5 & 5 & 6 \\
\hline
\end{tabular}

EMG was measured for five different positions including high (H-A), middle (H-B), low (H-C), lifting task, and normal standing posture. Overall, the low back and shoulders were commonly used for hand picking, while all muscles were used for lifting, as shown in Figure 5. The lifting task required the greatest muscle activity. For the harvesting task, the middle position required muscle activity like that of the standing position, resulting in similar EMG values between these two positions. The high position produced muscle activity three times higher than that of the standing posture. These results were consistent with the RULA postural analysis. 


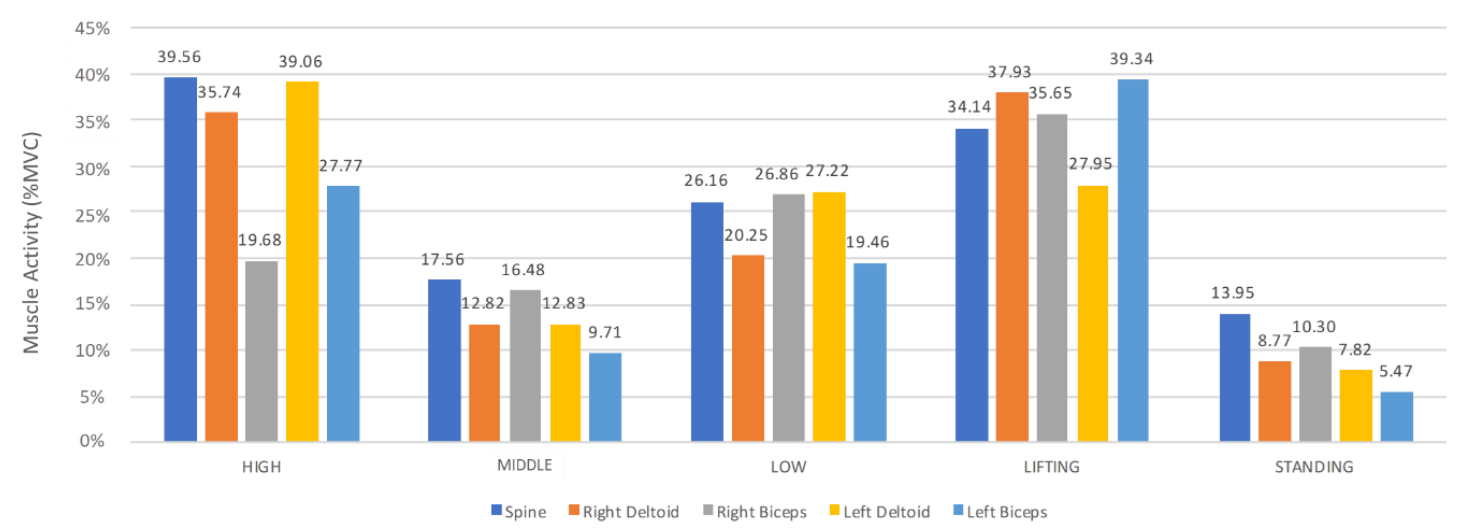

Figure 5. Muscle activity of five body regions by posture.

The mean Borg scale scores of the workers' perceived rate of discomfort is shown in Figure 6. The Borg scale scores showed a similar trend to that of the RULA analysis and EMG results. The low back exhibited the greatest mean Borg scale score (3.8), followed by the shoulder (3.5), neck (3.3), arm (3), and hand (2.7).

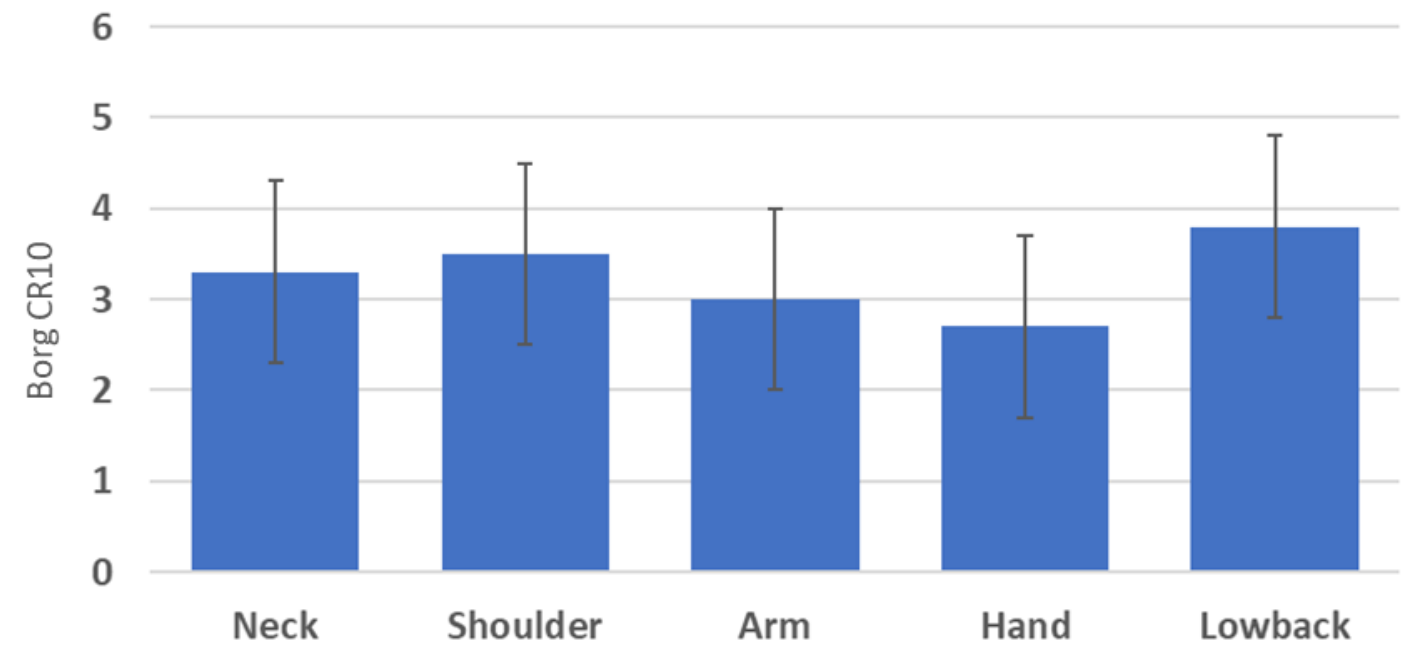

Figure 6. Subjective workload rating (Borg CR10).

Examples of the lifting postures are shown in Figure 7 and the results of the NIOSH lifting equations are shown in Table 4. The lifting task of picking up a bucket from the ground resulted in an $L I$ value greater that in the NIOSH lifting equation, indicating that this lifting posture may increase the risk of developing lifting-related low back pain. This high $L I$ value was due to the VM (Vertical Multiplier factor) since the worker needed to bend their trunk and knees to pick up the bucket from the ground without the aid of a raised pallet, which may contribute to low back pain. 


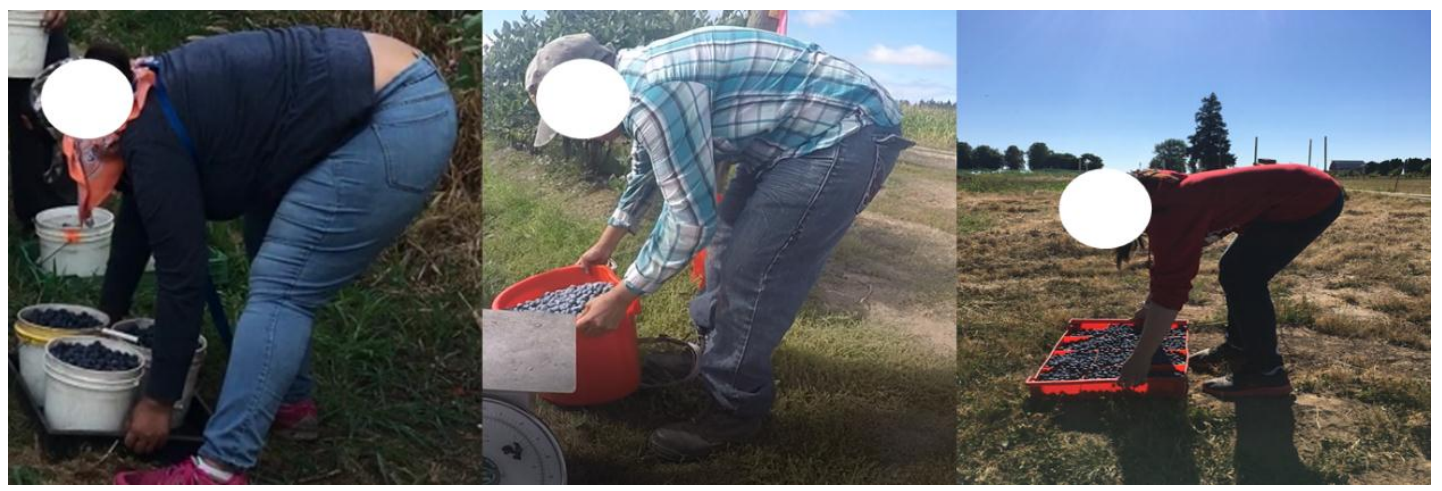

Figure 7. Example of lifting posture during hand harvesting.

Table 4. NIOSH lifting equation results for lifting task show in Figure 7.

\begin{tabular}{cc}
\hline Results & Values \\
\hline Recommend Weight Limit (RWL) & $5.56 \mathrm{~kg}$ \\
Lifting Index $(L I)$ & 1.26 \\
\hline
\end{tabular}

\subsection{Semi-Machine Harvesting with Hand-Held Shakers}

For semi-mechanical harvesting with hand-held power shakers, the result of CTD risk assessment for all postures was less than 1, indicating an acceptable risk of CTD for all tasks in the semi-machine harvesting process. Even though the posture factors exceeded the safety threshold of 1.0 with a mean value of 1.1 (SD: 0.14), the frequency factors were below the safety threshold of 1.0 with a mean value of 0.67 (SD: 0.07). Therefore, the total risk value was 0.73 (SD: 0.07). Table 5 shows the RULA scoring for semi-machine blueberry harvesting. Postures M-A and M-D each exhibited the highest possible RULA score of 7, mostly due to a side-bend of the trunk and an abducted shoulder posture. Since all postures showed a RULA score greater than 3, the machine design and/or worker posture should be adjusted to reduce or eliminate MSD risk.

Table 5. RULA analysis results.

\begin{tabular}{ccccc}
\hline Posture & Component Ratio (\%) & Score A & Score B & Grand Score \\
\hline M-A & 26 & 5 & 6 & 7 \\
M-B & 26 & 4 & 4 & 4 \\
M-C & 27 & 6 & 4 & 6 \\
M-D & 11 & 6 & 6 & 7 \\
M-E & 10 & 4 & 4 & 4 \\
\hline
\end{tabular}

For semi-machine harvesting with hand-held shakers, EMG values were measured for two positions: high (M-A and M-B) and low (M-C, M-D, and M-E). EMG results showed that both the high and low positions commonly required the right bicep to control the shaker, as shown in Figure 8 . The low position required the left deltoid to push and pull the shaker, while the high position required the right deltoid to control the shaker and the left bicep to push and pull the shaker. Since the low posture closely resembled standing posture, low back EMG values showed little difference between high and low postures. 


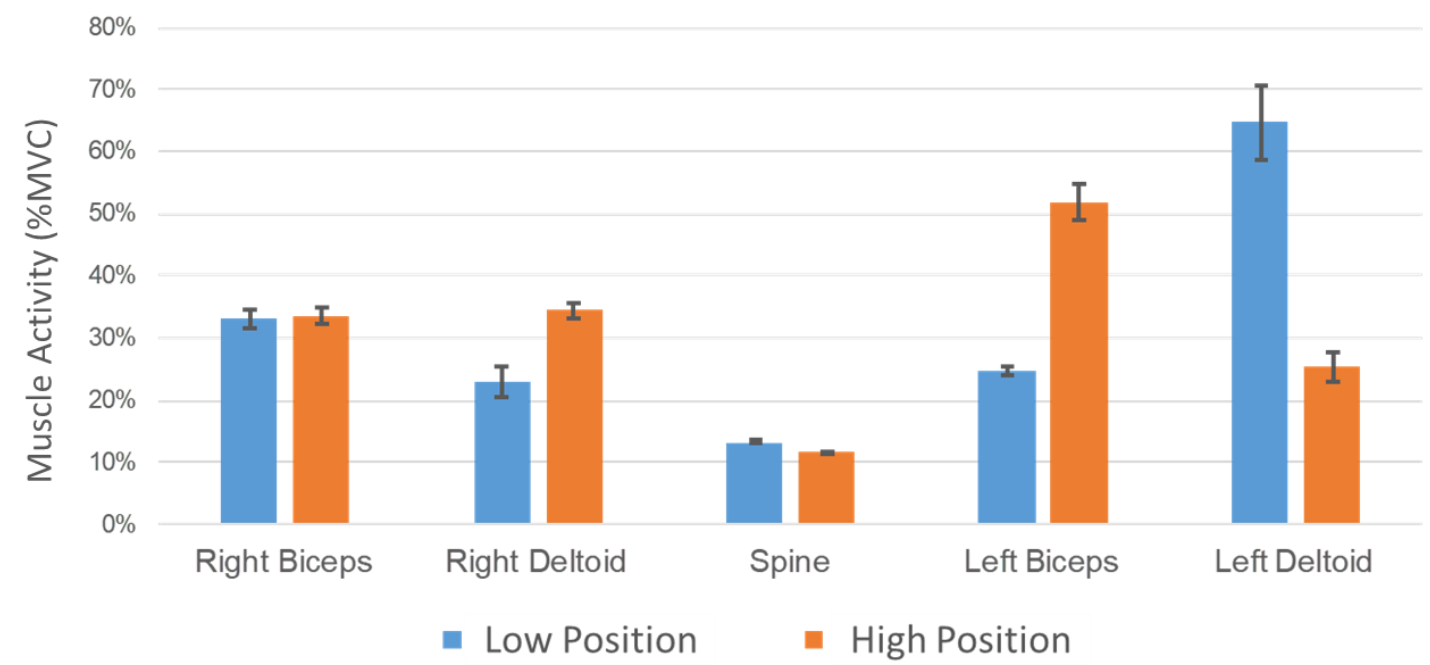

Figure 8. Muscle activity of five body regions by posture.

Example of the lifting posture are shown in Figure 9, with workers handling flats being filled with blueberries by an Oxbo 7240 harvester. The results of the NIOSH lifting equation are shown in Table 6. The lifting task at both origin and destination showed NIOSH equation values of less than 1 , indicating a nominal risk to healthy employees.

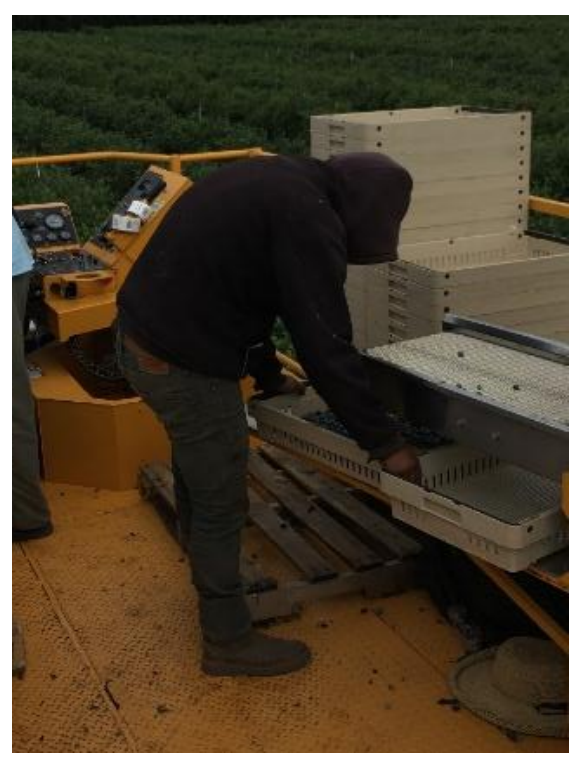

Figure 9. Example of lifting posture for handling flats filled with blueberries on an Oxbo 7240 blueberry harvester.

Table 6. NIOSH lifting equation results for lifting task.

\begin{tabular}{cc}
\hline Results & Values \\
\hline Recommend Weight Limit (RWL) & $11.88 \mathrm{~kg}$ \\
Lifting Index $(L I)$ & 0.80 \\
\hline
\end{tabular}

\subsection{OTR Machines}

The lifting of full lugs weighing $8.2 \mathrm{~kg}$ (18 lb.) was studied in workers operating the Oxbo 7440 and the Oxbo 8040 machines. An example of the lifting posture is shown in Figure 10 and the results of the NIOSH lifting equations are shown in Table 7. The Oxbo 7440 could be unloaded either from the 
back of the conveyor system or from the side. The same was the case for the Oxbo 8040. In all cases the NIOSH lifting index $(L I)$ was less than 1 , and thus acceptable for an 8-hour day.

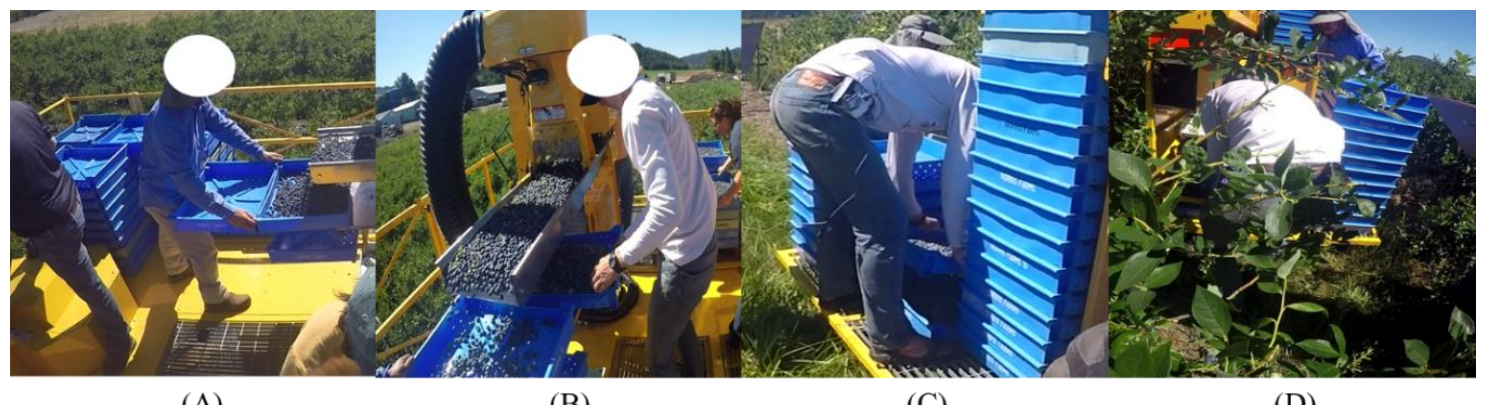

(A)

(B)

(C)

(D)

Figure 10. Example lifting postures: (A) Oxbo 7240 Back, (B) Oxbo 7440 Side, (C) Oxbo 8040 Side, and (D) Oxbo 8040 Back.

Table 7. National Institute for Occupational Safety and Health (NIOSH) lifting equation results for lifting task during over-the-row (OTR) harvesting.

\begin{tabular}{ccc}
\hline \multirow{2}{*}{ Oxbo 7440 Back } & Recommend Weight Limit (RWL) & $9.52 \mathrm{~kg}$ \\
& Lifting Index (LI) & 0.14 \\
\hline \multirow{2}{*}{ Oxbo 7440 Side } & Recommend Weight Limit (RWL) & $11.80 \mathrm{~kg}$ \\
& Lifting Index (LI) & 0.69 \\
\hline \multirow{2}{*}{ Oxbo 8040 Side } & Recommend Weight Limit (RWL) & $9.12 \mathrm{~kg}$ \\
& Lifting Index (LI) & 0.89 \\
\hline \multirow{2}{*}{ Oxbo 8040 Back } & Recommend Weight Limit (RWL) & $12.7 \mathrm{~kg}$ \\
& Lifting Index (LI) & 0.64 \\
\hline
\end{tabular}

EMG data is shown with respect to working conditions in Figure 11 and with respect to specific muscles in Figure 12. The Oxbo 8040 showed the worst EMG values with respect to working conditions, but the peaks (slightly over 40\% MVC) were only for short periods of time. Greatest muscle loading was as expected for the arms and shoulders, but because peak values marginally exceeded 40\% MVC and only for short periods of time, the loading posture associated with OTR machines was acceptable for an 8-hour day.

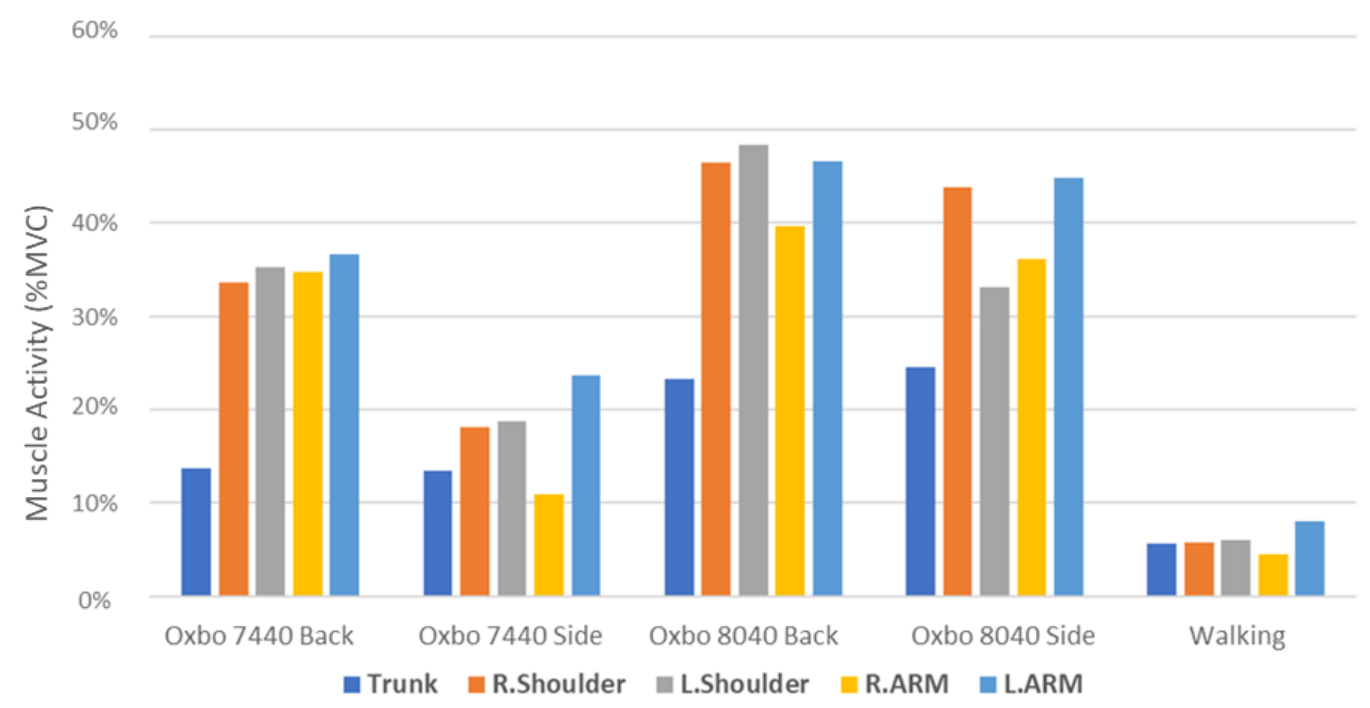

Figure 11. Muscle activity of five body regions by working condition (R.: Right; L.: Left). 


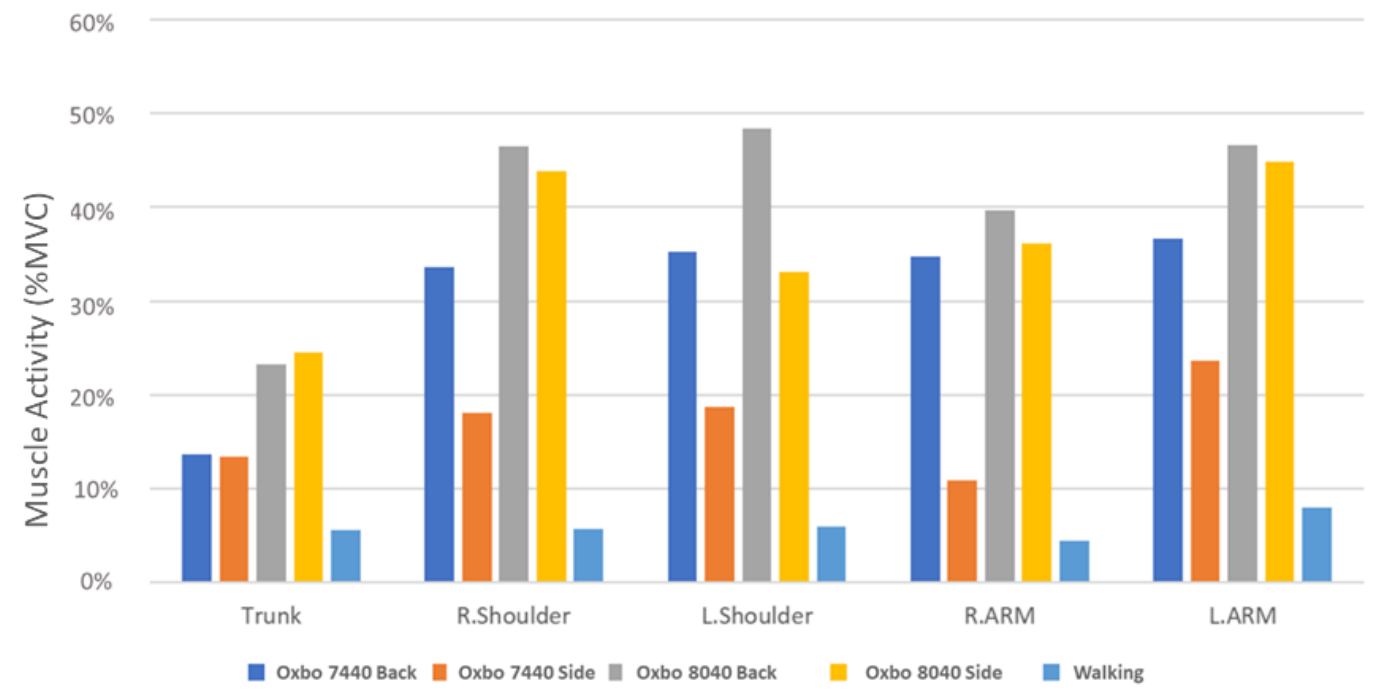

Figure 12. Muscle activity of five body regions by muscle (R.: Right; L.: Left).

\section{Discussion}

In this study, we conducted field research at several sites to investigate the risk factors of MSDs for each type of blueberry harvesting in terms of workers' postural loads and self-reported discomfort using ergonomics intervention techniques. Hand harvesting and semi-machine harvesting with hand-held shakers are difficult to analyze for fatigue, as there are many approaches with no clear limits. RULA values for hand picking ranged from 3 in the middle posture to 6 and 7 for low and high postures, respectively. Because the component ratio was over $50 \%$ in high and stretch position (H-A and $\mathrm{H}-\mathrm{D})$, we can conclude that this working posture contributes to workers' shoulder pain. The high scores of H-A and H-D suggest a need for redesign, but there is not much that can be done with hand harvesting. In addition, the RULA measurement assesses posture, but offers no clear insights on fatigue.

For semi-machine harvesting with hand-held shakers, the RULA numbers were also high, coming in between 4 and 7 , but this was due to excessive repetition of the reaching motion. Once the standing positions on the vehicle and the length of shaker are adjusted, then working posture and RULA scores will improve. The EMG value of various muscles indicate the forces utilized. For hand picking, EMG values for the high posture slightly exceeded $40 \% \mathrm{MVC}$, which is higher than the recommended $15 \%$ for static contractions. The shakers required 30-50\% MVC, but because this work is performed dynamically rather than in a static position, the recommended value of $15 \%$ MVC does not apply.

The best solution is to consider the Threshold Value Level [30] approach in conjunction with the Hand Activity Level (HAL). Two factors, force and HAL, determine whether the TLV is acceptable. The red region shown in Figure 13 represents hand harvesting, which has very high hand activity levels and lower EMG values but results in unacceptable fatigue values. The green region in Figure 13 represents the shakers, which have medium frequency and slightly higher EMG values but overall are still in the acceptable region for fatigue. The biggest problem with the shakers is the vibration, which can lead to fatigue and various risks for workers, especially in the upper limbs. Reducing the vibration with gel pads or gloves would decrease the risk to workers, as found in 2016 in the North Carolina studies that used simple foam as a stop-gap approach. However, according to a previous study [31], since anti-vibration gloves only extend the vibration exposure over time and therefore do not completely prevent the wearer from developing Hand-Arm Vibration Syndrome (HAVS), more research is required to conduct ergonomic interventions with reliable vibration data, not only with that obtained by subjective perceptions. In addition, according to a previous study conducted by Takeda et al. [11], hand-held shaker devices have not been widely adopted among blueberry growers due to harvest inefficiency and fatigue after prolonged use. Furthermore, Calvo et al. [32] pointed 
out that vibrating tools used in manual olive harvesting exceed the admitted limits of occupational repetitive action (OCRA) scores. Thus, workers who use vibrating tools in unnatural body postures are at risk for disorders of the upper limbs.

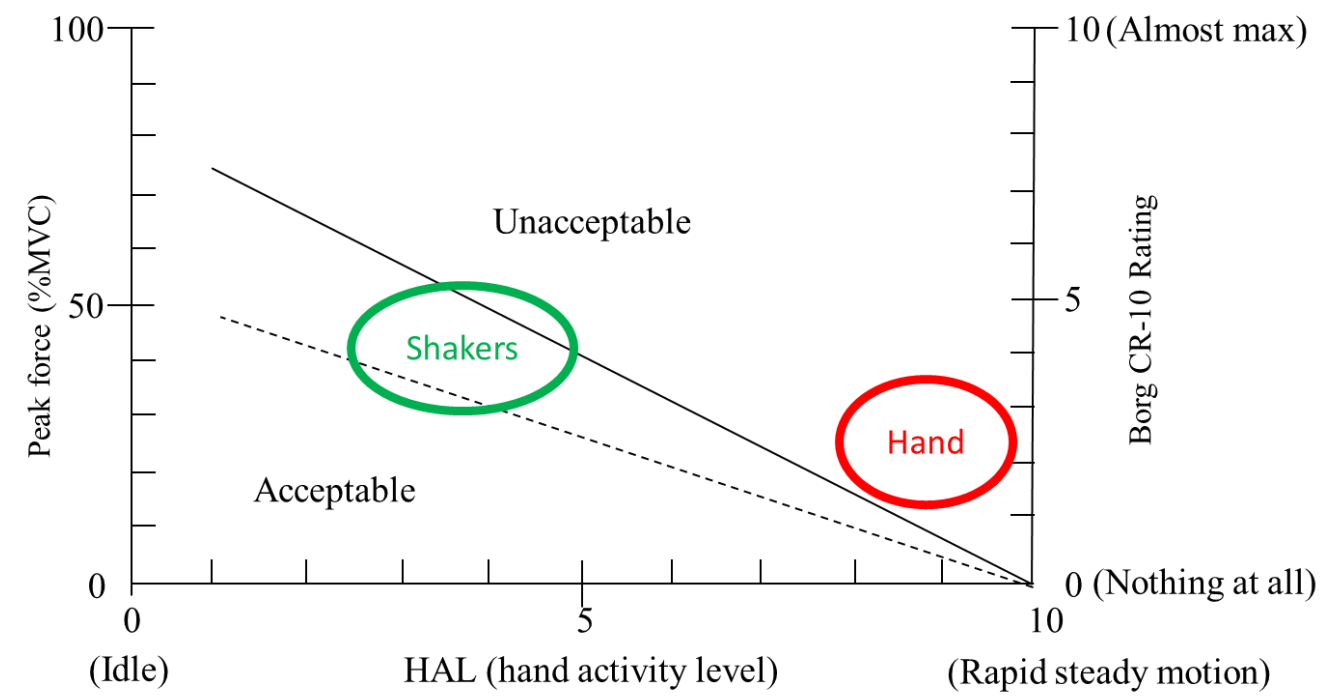

Figure 13. Threshold Level Values for Shakers and Hand Harvesting.

OTR harvesting eliminates repetitive hand motions and transfers all the work to simple lifting, for which there are clear approaches, such as the NIOSH lifting guidelines, to be used. In all cases, the lifting index was below 1 and therefore acceptable for an 8-hour day. This indicates that the automated harvester would be the best approach in minimizing worker loading and fatigue. However, it would be challenging for small- and medium-sized blueberry farms to purchase automated harvesters due to their high cost. Thus, collaborative efforts among health and safety professionals, engineers, social scientists, and ergonomists are needed to provide effective ergonomic interventions, including mechanical worker aid devices and tools as well as engineering and administrative controls such as programmed rest breaks, job rotation, and worker training.

Our study presents some features and limitations that should be noted. The first limitation of this study is the limited data to obtain reliable demographic results. Since most harvesters were migrants, the manager did not allow the collection of demographic data. The second limitation of this study is that it does not provide a solution that would eliminate risk factors of MSDs for hand and semi-machine harvesting. For example, this study does not suggest how to redesign the shaker to reduce vibration problems or how to train workers to adopt proper harvesting posture. However, the purpose of this study was to identify the risk factors of each type of blueberry harvesting. Thus, this limitation should not change the conclusions of this study. Further research will be required to find solutions for aid devices and tools as well as adequate training to reduce MSDs.

Author Contributions: E.K. wrote the manuscript. E.K. and A.F. collected and analyzed the data. F.T. and C.L designed and implemented the shakers and the mechanized harvester experiments. A.F. and F.T. edited the manuscript.

Funding: This research was funded by USDA National Institute of Food and Agriculture Specialty Crop Research Initiative, grant number 2014-51181-22383.

Conflicts of Interest: The authors declare no conflict of interest. 


\section{References}

1. Bureau of Labor Statistics (BLS). Occupational Injuries/Illnesses and Fatal Injuries Profiles; Department of Labor, Bureau of Labor Statistics: Washington, DC, USA, 2016.

2. Putz-Anderson, V.; Bernard, B.P.; Burt, S.E.; Cole, L.L.; Fairfield-Estill, C.; Fine, L.J.; Grant, K.A.; Gjessing, C.; Jenkins, L.; Hurrell, J.J., Jr.; et al. Musculoskeletal Disorders and Workplace Factors; National Institute for Occupational Safety and Health (NIOSH): Cincinnati, OH, USA, 1997; p. 104.

3. Whelan, S.; Ruane, D.; McNamara, J.; Kinsella, A.; McNamara, A. Disability on Irish farms-A real concern. J. Agromed. 2009, 14, 157-163. [CrossRef] [PubMed]

4. McMillan, M.; Trask, C.; Dosman, J.; Hagel, L.; Pickett, W.; for the Saskatchewan Farm Injury Cohort Study Team. Prevalence of Musculoskeletal Disorders among Saskatchewan Farmers. J. Agromed. 2015, 20, $292-301$. [CrossRef] [PubMed]

5. Walker-Bone, K.; Palmer, K.T. Musculoskeletal disorders in farmers and farm workers. Occup. Med. 2002, 52, 441-450. [CrossRef]

6. Fathallah, F.A. Musculoskeletal disorders in labor-intensive agriculture. Appl. Ergon. 2010, 41, 738-743. [CrossRef] [PubMed]

7. Choobineh, A.; Tabatabaee, S.H.; Behzadi, M. Musculoskeletal Problems among Workers of an Iranian Sugar-Producing Factory. Int. J. Occup. Saf. Ergon. 2009, 15, 419-424. [CrossRef] [PubMed]

8. Ng, Y.G.; Bahri, M.T.S.; Syah, M.Y.I.; Mori, I.; Hashim, Z. Ergonomics observation: Harvesting tasks at oil palm plantation. J. Occup. Health 2013, 55, 405-414. [CrossRef] [PubMed]

9. Kirkhorn, S.R.; Earle-Richardson, G.; Banks, R.J. Ergonomic risks and musculoskeletal disorders in production agriculture: Recommendations for effective research to practice. J. Agromed. 2010, 15, 281-299. [CrossRef] [PubMed]

10. Mora, D.C.; Miles, C.M.; Chen, H.; Quandt, S.A.; Summers, P.; Arcury, T.A. Prevalence of musculoskeletal disorders among immigrant Latino farmworkers and non-farmworkers in North Carolina. Arch. Environ. Occup. Health 2016, 71, 136-143. [CrossRef] [PubMed]

11. Takeda, F.; Yang, W.; Li, C.; Freivalds, A.; Sung, K.; Xu, R.; Hu, B.; Williamson, J.; Sargent, S. Applying New Technologies to Transform Blueberry Harvesting. Agronomy 2017, 7, 33. [CrossRef]

12. Brown, G.K.; Schulte, N.L.; Timm, E.J.; Beaudry, R.M.; Peterson, D.L.; Hancock, J.F.; Takeda, F. Estimates of mechanization effects on fresh blueberry quality. Appl. Eng. Agric. 1996, 12, 21-26. [CrossRef]

13. Mehra, L.K.; MacLean, D.D.; Savelle, A.T.; Scherm, H. Postharvest disease development on southern highbush blueberry fruit in relation to berry flesh type and harvest method. Plant Dis. 2013, 97, 213-221. [CrossRef]

14. Casamali, B.; Williamson, J.G.; Kovaleski, A.P.; Sargent, S.A.; Darnell, R.L. Mechanical Harvesting and Postharvest Storage of Two Southern Highbush Blueberry Cultivars Grafted onto Vaccinium arboreum Rootstocks. HortScience 2016, 51, 1503-1510. [CrossRef]

15. Lobos, G.A.; Moggia, C.; Retamales, J.B.; Sanchez, C. Effect of mechanized (self-propelled or shaker) vs. hand harvest on fruit quality of blueberries (Vaccinium corymbosum L.) in postharvest. In Proceedings of the X International Symposium on Vaccinium and Other Superfruits 1017, Maastricht, The Netherlands, 17-22 June 2012; pp. 141-145.

16. Borg, G. Psychophysical scaling with applications in physical work and the perception of exertion. Scand. J. Work Environ. Health 1990, 16, 55-58. [CrossRef] [PubMed]

17. Robertson, R.J.; Stanko, R.T.; Goss, F.L.; Spinal, R.J.; Reilly, J.J.; Greenawalt, K.D. Blood glucose extraction as a mediator of perceived exertion during prolonged exercise. Eur. J. Appl. Physiol. 1990, 61, 100-105. [CrossRef]

18. Gearhart, R.E.; Goss, F.L.; Lagally, K.M.; Jakicic, J.M.; Gallagher, J.; Robertson, R.J. Standardized scaling procedures for rating perceived exertion during resistance exercise. J. Strength Cond. Res. 2001, 15, 320-325. [PubMed]

19. Troiano, A.; Naddeo, F.; Sosso, E.; Camarota, G.; Merletti, R.; Mesin, L. Assessment of force and fatigue in isometric contractions of the upper trapezius muscle by surface EMG signal and perceived exertion scale. Gait Posture 2008, 28, 179-186. [CrossRef] [PubMed]

20. McAtamney, L.; Corlett, E.N. RULA: A survey method for the investigation of work-related upper limb disorders. Appl. Ergon. 1993, 24, 91-99. [CrossRef] 
21. Seth, V.; Lee Weston, R.; Freivalds, A. Development of a cumulative trauma disorder risk assessment model for the upper extremities. Int. J. Ind. Ergon. 1999, 23, 281-291. [CrossRef]

22. Freivalds, A. Niebel's Methods, Standards, and Work Design; Mcgraw-Hill Higher Education: Boston, MA, USA, 2009; Volume 700.

23. Waters, T.R.; Putz-Anderson, V.; Garg, A.; Fine, L.J. Revised NIOSH equation for the design and evaluation of manual lifting tasks. Ergonomics 1993, 36, 749-776. [CrossRef] [PubMed]

24. Garg, A.; Boda, S. The NIOSH Lifting Equation and Low-Back Pain, Part 1. Hum. Factors 2014, 56, 6-28. [CrossRef] [PubMed]

25. Waters, T.R.; Putz-Anderson, V.; Baron, S. Methods for assessing the physical demands of manual lifting: A review and case study from warehousing. Am. Ind. Hyg. Assoc. J. 1998, 59, 871-881. [CrossRef] [PubMed]

26. van der Beek, A.J.; Mathiassen, S.E.; Windhorst, J.; Burdorf, A. An evaluation of methods assessing the physical demands of manual lifting in scaffolding. Appl. Ergon. 2005, 36, 213-222. [CrossRef] [PubMed]

27. Kucera, K.L.; Loomis, D.; Lipscomb, H.J.; Marshall, S.W.; Mirka, G.A.; Daniels, J.L. Ergonomic risk factors for low back pain in North Carolina crab pot and gill net commercial fishermen. Am. J. Ind. Med. 2009, 52, 311-321. [CrossRef] [PubMed]

28. Lavender, S.A.; Oleske, D.M.; Nicholson, L.; Andersson, G.B.; Hahn, J. Comparison of five methods used to determine low back disorder risk in a manufacturing environment. Spine 1999, 24, 1441. [CrossRef] [PubMed]

29. Waters, T.R.; Lu, M.-L.; Piacitelli, L.A.; Werren, D.; Deddens, J.A. Efficacy of the revised NIOSH lifting equation to predict risk of low back pain due to manual lifting: Expanded cross-sectional analysis. J. Occup. Environ. Med. 2011, 53, 1061-1067. [CrossRef] [PubMed]

30. ACGIH. TLVs and BEIs Threshold Limit Values for Chemical Substances and Physical Agents and Biological Exposure Indices; American Conference of Governmental Industrial Hygienists: Cincinnati, OH, USA, 2002.

31. Sampson, E.; Van Niekerk, J.L. Literature Survey on Anti-Vibration Gloves. 2003. Available online: https:/ / scholar.google.com/scholar?hl=en\&q=Literature+survey+on+anti-vibration+gloves\&btnG= \&as_sdt=1\%2C39\&as_sdtp $=($ accessed on 23 October 2018).

32. Calvo, A.; Romano, E.; Preti, C.; Schillaci, G.; Deboli, R. Upper limb disorders and hand-arm vibration risks with hand-held olive beaters. Int. J. Ind. Ergon. 2018, 65, 36-45. [CrossRef] 